\title{
Developing a facilities management questionnaire in a local government setting
}

Daniel Adler

City of Sydney

\begin{abstract}
Community facilities are at the frontline of service provision for local government, enacting strategy and objectives on a day-to-day basis and forming the cornerstone of performance assessment. The performance of facilities, as judged by their users, is a contested area of governance, with theories on assessment being influenced by economic, social, environmental and business-as-usual advocates. Facility managers, who see users as the source of their reward as well as their day-to-day problems, consider asking users for their opinion as being equally a benefit and a risk. However, measuring customer satisfaction is increasingly emerging as a measure of good performance and facility managers have to find some credible way of gauging this. This research paper takes a broadly consultative approach to survey development and finds that, regardless of the best planning and design, facility managers will be judged based on the limited experiences of their users and evaluators and this is key to successfully embedding new strategy in operations.
\end{abstract}

Key words: community; questionnaire; customer; facilities; stakeholder; accessibility

\section{Introduction}

This research paper explores ways of constructing customer satisfaction questionnaires in community facilities in a local government setting. The main theme explored is the concept of measuring how the facility management practices and customer service provided impact upon accessibility to the facilities for the members of the community who use them. This will be explored through a review of the relevant literature, and by using the City of Sydney (CoS) Community Venues (Facilities) as an example of questionnaire development through consultation with the facility hirers, community facility management, and project coordinators responsible for organizing community-based programs in the facilities.

The CoS provides a range of Community Venues (Facilities) that are used for a variety of programs and activities run by groups including local council, government agencies, nongovernment organizations (NGOs), commercial organizations, private individuals, combinations of the above and others. These facilities are provided in a do-it-yourself style, with hirers required to administer their own access, room setups, room breakdowns and room cleanups. The $\mathrm{CoS}$ Community Venues (Facilities) are managed by three general areas. The CoS Property Unit owns and maintains the physical spaces through an ongoing cleaning, maintenance, and renovation program. The spaces are used and venues managed with the assistance of Community Venues staff by local council, government agencies, NGOs, commercial organizations, private individuals who deliver a variety of programs and activities ranging from (but not limited to) council meetings, NGO training days, fundraisers, sales and family functions. The day-to-day use of the spaces is coordinated by the CoS Community Venues (Facilities), a facility services 
team providing scheduling, cleaning and maintenance inspections, billing/insurance/contracts, and other customer service and venue management-related functions.

The challenge for the CoS is to match the rhetoric of its "Sustainable Sydney 2030" strategic plan with on-the-ground results. In the case of community facilities, how will the organization know it is achieving strategy? The key is having an agreed approach to occupant feedback that aligns with all stakeholders' objectives, and is flexible through time to adapt to changing environments. This paper is an attempt to bring all stakeholders' perspectives into one feedback mechanism and provide a template for ongoing feedback development.

\section{Literature review}

A review of the relevant literature highlights four key areas that contribute to best practice in user satisfaction in community facilities management: engaging stakeholders, post-occupancy evaluation, benchmarking and integration with other public services. Measuring customer satisfaction is widely acknowledged as best practice across industries and the use of questionnaires is recognized as a valuable and low-cost method of collecting information from broad representations of customers to verify whether business operations and projects are delivering the strategic objectives of the organization (Bryman \& Cramer 1990; Hair et al. 2007).

Engaging stakeholders is used as a design process for determining who will use the space, and the various requirements of the users in regard to their ability to act in the space. McShane (2006) argues that the three most important areas are "ownership, change management and governance". The author focuses on the renewal and development of community facilities in local government in Australia and the challenges of balancing competing goals. McShane discusses the history of facility management and identifies challenges in development in facility operation and community engagement. However, there is no discussion on current and future operations management of the facilities.

Church and Marston's (2003) study into activity-based modeling uses measures such as time and distance to determine if users can use space effectively as it relates to their external activities. They discuss issues relating to activities rather than structures; for example, how long does it takes to get from the office to the coffee cart if you are unable to use the stairs, and how does this relate to other social activity? Their result is a series of algorithms to measure how environments perform based on multiple activities that might be performed there by people with different activity types. This is relevant to community facilities as they provide services to many different people with varied access requirements and capabilities. This also has implications on timing of activities and how long it takes people to attend the facilities. Internal to the facilities, there are additional implications on the choice of furniture and the location of amenities on site as the time it takes to prepare a room can influence choices around usage.

Another example of this is Dong et al.'s (2006) research into “Activity Based Accessibility". This measure seeks to go from measuring activities in isolation to a model called the "Day activity schedule". This takes a log of all activities in a day, measures how long they take, compares them to the desired activities of a given individual and indicates where the barriers or inefficiencies are. It is an efficiency measure that can be used to identify overlapping patterns of access barriers that limit the quality and quantity of activities an individual can undertake on any 
given day based on their (dis)location from services, and the places, services and people they need to visit. This is applicable to users of community facilities as they need to have a range of appropriately located and fitted out facilities located within their possible range of periodic activity. More significantly, this raises the question: are there barriers in the community facilities experience that reduce the overall access to the community services at other localities and times for the individuals who attend them?

Post Occupancy Evaluation is also used as a method for evaluating the quality of customer services provided across a range of industries (Reichheld 2003). Here a simple reductionist approach to evaluation is put forward and the only business evaluation process necessary are the questions, "would you recommend us to friends and colleagues?" and "would you buy from us again?" - the idea being that word of mouth from loyal customers is of more value in attracting new customers. This has relevance to community facilities in regard to keeping loyal customers happy. However the author's field of reference is for profit enterprise and there is no discussion of other ways of service interaction. Opposing views that look at the issue from a not-for-profit organization perspective argue that harsh economic bottom lines as discussed by Reichheld are counterproductive in practice. Evans (2000) discusses the impact of the triple bottom line (economy, efficiency and effectiveness) on arts organizations in the United Kingdom over a 10year period, which indicates that arts organizations are losing their identity and are being viewed more as consumer goods and losing their intrinsic value as cultural treasures. The visible result is a decline in attendance even though efficiency (as measured by the triple bottom line) has increased. This is important to community facilities as they are providing a not-for-profit service to the community and therefore may want to avoid the performance measures of for-profit business. The implication for a community facilities questionnaire is that questions will need to be framed to evaluate the facilities against the organizational vision and strategic objectives rather than externally generated frames of reference such as the triple bottom line. Kincaid (1994) gives an example of developing this type of locally generated performance measure, where measuring the performance of facilities management through Post Occupancy Evaluation is discussed. Kincaid argues that feedback from users of facilities in the planning and strategy of facility management is important. The author suggests a method of valuing the requirement of the service versus the quality of service provided. The author also draws attention to the risk of customer engagement being the expectation of resultant action.

Benchmarking against other facilities is also a popular theme in determining the quality of facilities provided. Brackertz and Kenley (2002) discuss "logometrix" and "service balanced scorecard" as essential elements to benchmark facilities management of community facilities operated by councils within and across councils. Of particular interest to customer satisfaction questionnaires are a set of indicators or the "7 elements" that form part of the "Service Indicator" out of which questions can be asked: transport accessibility, safety, location, disability access, equity, design/fit-out, and building functionality. These elements are scored by "functional requirement" and then "actual performance" using a Likert-type scale. There are two aspects of information required: how users rate the importance of the provision of a specific element for the activity they need to undertake at a community facility versus how they rate the performance of those weighted specifications as they are provided. This can be used to prioritize upgrades to service delivery as well as give feedback on the community's perceptions of council strategies. Brackertz (2006) further develops this research through exploring the correlation between physical and service performance of community facilities in two councils. The paper studies the 
implementation of a facilities management tool called logometrix in two councils in Victoria, Australia. It compares the results of each council to see if there is a dependent relationship between building quality and service provision. The interesting point here is that in council A the model was rolled out by the facilities management branch (engineering and construction) but in council B it was rolled out by the social policy and community support branch. The influence and understanding of good facility management seemed to significantly influence the results. This could be an indication of a shortfall in the logometrix as the business unit which is in control of the roll-out seems to have influence on the outcome.

\section{Integration with other public services}

Weber and Khan (2002) discuss at a broad level the linking of community need to facility management by local government in Victoria. The authors' identify challenges around appropriate consultation and the need to integrate facilities into the community. The authors' focus on the apparent need to renovate and redevelop facilities and the challenges this will bring to community cohesion. There is little discussion on the specifics of engaging the community other than criticisms of "town hall" meetings as being a token measure by which local government claim community engagement. The paper informs the community facilities survey by placing the issues in the broader context and identifying the problems; however, it does not provide any demonstrated solutions. This issue is also researched in the work of Besnard and Council (2002) around value management in community facilities managed by local government and place facility management in the context of being a delegated responsibility from state government with the local authority responsible for the actual implementation of facility management to the community. Wollongong Council is the case study and the development of its flagship community facility the object. This paper falls into the same ethos of using redevelopment as a tool to manage ongoing problems. The logic here is that built environment rather than community engagement is the solution.

In summary, the literature reviewed above suggests that questionnaire development should be targeted to identified relevant populations and involve relevant stakeholders to ensure the appropriate questions are asked. Further, in order to embed development in community participation there must be an inbuilt feedback mechanism whereby the customer or hirer of the facility can have some control over venue management and the customer service provided that goes beyond their purchasing and voting power.

\section{Methodology}

Through extensive consultation with stakeholders the method used to determine appropriate questions involved the combination of the following seven areas:

1. Review of existing surveys within the $\mathrm{CoS}$ was identified as a valuable resource due to the surveys already being tested on populations using the facilities and other CoS services. Questionnaires were requested from related business units such as Youth Services, Aged and Disability Services, Library and Cultural Services, Commercial Facility Management, Community Centre, Customer Service, and Brookefield Multiplex (the contracted facility maintenance provider). 
2. Informal discussions with project coordinators who organize program delivery in the facilities as well as advise on social policy issues related to the groups who hire the facilities was also identified as a valuable resource as they would be able to advise on how to engage specific $\mathrm{CoS}$ target populations of hirers such as CALD, Aboriginal and Torres Strait Islander, Public Housing, Youth and Disability.

3. Consultation with community facilities management to determine the relevant business processes to evaluate and to refine the language, terminology, layout and purpose of the questionnaire.

4. Based on the relevant literature in the area discussed in the literature review above, the main themes of accessibility, value for money, safety, cleanliness, maintenance and usability of facilities were included in the questionnaire.

5. A pilot of the survey with selected hirers to evaluate quality in terms of ease in answering, and whether the questions covered the concerns of the hirers. A mix of council, NGO, and commercial hirers were selected to evaluate the survey.

6. Peer review of the questionnaire development and design was conducted weekly within the University of Technology Sydney (UTS) Industry Based Project Studies online forum.

7. A review of relevant $\mathrm{CoS}$ policies and guidelines regarding the provision of community facilities including the CoS Vision contained in the 2030 document, the Corporate Plan, and the Social Policy and Social Plan. 